\title{
Francis Blache et la fabrication des sous-produits de la houille : entre veille et expérimentation
}

\author{
Francis Blache and the manufacture of coal by-products: between \\ intelligence technology and experimentation
}

\author{
Luc Rojas $^{1}$ \\ ${ }^{1}$ UMR CNRS 5600 Universités de Lyon - Université de Saint-Étienne (luc.rojas@univ-st-etienne.fr)
}

\begin{abstract}
RÉSUMÉ. A l'instar des autres charbonnages, les mines de Montrambert sont confrontées à une crise industrielle à la fin du XIXe siècle les mettant en difficulté sur certains marchés. Pour répondre à cela, la houillère souhaite mettre en valeur l'ensemble de ses houilles et notamment les déchets de celles-ci. Elle confie cette mission à Francis Blache : un ingénieur qui prend la tête des usines d'essai édifiées pour l'occasion. Pour satisfaire les exigences de la compagnie, il fait le choix de l'alliance entre la science et l'industrie et ouvre au charbonnage de nouveaux marchés notamment celui du gaz.

ABSTRACT. Like other collieries, Montrambert experienced difficulties with the economic markets at the end of the 19th century. To fight against these difficulties, the colliery wanted to exploit coal waste. The company entrusted this mission to Francis Blache. The engineer became factory manager: he chose to combine science and industry. His choices enabled the company to obtain new markets, notably that of gas.

MOTS-CLÉS. Charbon, expérimentation, veille technologique, ingénieur civil, pratique technique.

KEYWORDS. Coal, experimentation, intelligence technology, civil engineer, technical practice.
\end{abstract}

Tout au long du XIX ${ }^{\mathrm{e}}$ siècle le charbon de terre se place au cœur du système énergétique devenant le pain de l'industrie. Les charbonnages connaissent une expansion remarquable en décuplant rapidement leurs productions et leurs effectifs ouvriers [VEY 99]. Durant les deux premiers tiers du siècle, les compagnies houillères explorent leurs gîtes afin d'augmenter le tonnage pouvant être mis à la disposition des différentes industries. Il faut attendre les années 1880 et la grande crise métallurgique pour que les charbonnages développent massivement des procédés techniques permettant de fabriquer des sous-produits issus de la houille afin de diversifier leurs clientèles. Ainsi ces entreprises font face à une crise qui, entre 1873 et 1896, engendre une baisse des prix et un ralentissement de la production et des échanges à l'échelle mondiale [WOR 98].

La diversification des usages du charbon apparaît aussi comme une réponse adaptée à la demande sociale. En effet, à la Belle époque la houille commence à être utilisée pour produire du gaz et de l'électricité : 5\% du charbon est destiné à cet usage en Allemagne en 1909, 7\% en France en 1913 et $12 \%$ au Royaume-Uni en 1913. Les foyers profitent de cela car les réchauds et les cuisinières à gaz sont devenus d'un usage courant au début du $\mathrm{XX}^{\mathrm{e}}$ siècle. A cette époque, dans les villes disposant de réseaux de distribution, ils représentent une consommation à peu près équivalente à celle de l'éclairage [CAR 97].

Néanmoins, les houillères ne connaissent pas un développement identique car elles restent soumises aux caractéristiques géologiques de leur gîte. Ainsi, certaines compagnies possèdent des qualités de charbons très différentes pouvant s'adapter aux marchés métallurgiques, du chauffage domestique ou encore des chemins de fer. A la fin du $\mathrm{XIX}^{\mathrm{e}}$ et au début du $\mathrm{XX}^{\mathrm{e}}$ siècle l'innovation devient une réponse permettant, pour les charbonnages, de mettre en valeur l'ensemble des houilles présentes sur leur territoire et d'accéder ainsi à de nouveaux marchés.

En France, à l'ouest de Saint-Étienne, les houillères de Montrambert et de la Béraudière exploitent un gisement, depuis 1854, reconnu pour la qualité de ses charbons ne nécessitant que peu 
d'intervention (lavage, concassage...) avant d'être vendus [ROJ 17]. Néanmoins, cette compagnie subit également les effets de la crise métallurgique et se voit dans l'obligation de développer le traitement des charbons afin d'accéder à de nouveaux marchés et d'augmenter ses bénéfices pour compenser la perte de certains marchés. Cette tâche essentielle au développement de l'entreprise est confiée à Francis Blache, ingénieur issu de l'Ecole des Mines de Saint-Étienne. Plus globalement, le cas de cet ingénieur et de sa mission interroge le positionnement de l'innovateur au sein de l'entreprise : à quelle demande doit-il répondre ? Quelles exigences doit-il satisfaire ? Est-il le seul décisionnaire dans le processus d'innovation qu'il est sensé piloter ? L'exemple de Francis Blache nous donne également à voir ce que Bergson nomme la latitude de création [BER 07], c'est à dire la stratégie développée par l'innovateur pour atteindre les objectifs qui lui sont fixés par sa hiérarchie. Autrement, dit l'ingénieur se doit de développer une intelligence technique capable de prendre en compte les différentes contraintes qu'il rencontre, qu'elles soient économiques, géologiques, industrielles ou autres, afin de mener à bien sa mission.

Mener à bien une telle ambition nécessite des sources nous entraînant dans l'activité quotidienne de l'ingénieur, dans sa pratique professionnelle et industrielle. Les archives des houillères de Montrambert et de la Béraudière conservées aux Archives départementales de la Loire nous offrent cette possibilité et nous permettent également de confronter la pratique de l'ingénieur avec les desideratas et les impératifs économiques du charbonnage. Afin de lever le voile sur ces interrogations, nous analyserons la volonté des houillères de Montrambert et de la Béraudière d'investir de nouveaux marchés. Puis nous nous pencherons sur le processus d'expérimentation mis en place par Francis Blache au sein des usines de la compagnie. Enfin nous achèverons notre texte en portant un regard sur l'activité industrielle des usines dirigées par Blache.

\section{Répondre à la volonté de la compagnie : investir de nouveaux marchés en valorisant toutes les houilles}

\subsection{Des effets de la crise métallurgique.....}

La crise métallurgique apparaissant dans les années 1880 affecte de nombreux domaines industriels. Ainsi, l'industrie de la locomotive connaît la récession entraînant l'effondrement de la fourniture de rails qui passe de 410000 tonnes en 1883 à 169000 tonnes en 1889. Le bassin stéphanois est touché de plein fouet. Les productions de fonte et d'acier reculent respectivement des deux tiers et de moitié entre 1880 et 1895 [WOR 98]. Dans le même temps l'apparition du procédé Thomas et Gilchrist permet à la Lorraine française d'exploiter les abondants gisements de fer phosphoreux avec un prix de revient très bas provoquant la fin de la fabrication des rails dans les usines stéphanoises. La crise que traverse la métallurgie stéphanoise amène au début des années 1880 à la liquidation de l'importante société de Terrenoire, La Voulte et Bessèges. C'est notamment à partir de 1884 que la crise se manifeste dans l'ensemble de la production métallurgique. Les entreprises se spécialisent et diminuent leur production de métal [GRA 08].

Cette diminution est donc la conséquence de la concurrence lorraine mais aussi celle de la concurrence littorale (près de Nantes, de Bayonne et dans le Nord) approvisionnée en minerai étranger [WOR 98]. Ceci affecte indirectement les houillères de la région stéphanoise qui accusent, en 1884, une baisse de la production de près de 400000 tonnes. A l'instar de certaines entreprises métallurgiques, la crise amène à la faillite la compagnie des mines de la Chazotte. L'ensemble des prix des charbons menus et du coke destinés à la métallurgie sont en baisse [GRA 22]. Malgré un léger rebond de la production à la fin des années 1880, les houillères stéphanoises connaissent de nouveau, à partir de 1891, une diminution de la production. Celle-ci se confirme en 1894. Les causes sont diverses : des hivers très doux qui ne facilitent pas la vente du charbon destiné au chauffage domestique, le refoulement des charbons stéphanois par la baisse du fret de l'Angleterre sur Marseille, la concurrence des prix des houillères allemandes sur le marché suisse.... Au-delà de la diminution de la production, les charbonnages stéphanois subissent également une baisse de leur 
prix de vente de près de 1 franc par tonne. Ce sont les cokes destinés à la métallurgie qui connaissent la chute la plus notable, la production passant de 116000 tonnes en 1893 à 86000 tonnes en 1894 soit une chute de près de $25 \%$ [GRA 22].

Ainsi, le ralentissement de la métallurgie et des autres activités industrielles engendrent presque automatiquement une diminution de la production des houillères. La compagnie des mines de Montrambert et de la Béraudière n'échappe pas à la règle même si parfois cette crise s'exprime différemment dans son activité. Le conseil d'administration note lors de l'assemblée générale de 1883 que la vente des charbons a augmenté alors que l'activité métallurgique est en régression dans la région stéphanoise. Ce conseil veut y voir une preuve du développement des autres usages du charbon ${ }^{1}$. En réalité, la crise ne touche le charbonnage que quelques années plus tard car Montrambert ne fournit pas les usines produisant les rails nécessaires au chemin de fer. Il faut attendre 1886 pour que les effets atteignent la houillère et modifient l'attitude de la clientèle qui recherche désormais des houilles bon marché et de qualités inférieures ${ }^{2}$. Montrambert souffre de l'enclavement du bassin stéphanois qui augmente considérablement le prix de revient [ROJ 08] et de la qualité supérieure de ses charbons de forge. Ainsi le charbonnage entre véritablement dans cette crise métallurgique en 1886. Cela se confirme tout au long de la décennie 1890 avec l'apparition d'une concurrence nouvelle. A l'instar des autres houillères, les concurrences lorraine et littorale jouent un rôle non négligeable mais Montrambert doit également composer avec le développement de l'emploi des anthracites dans les phares et l'éclairage électrique qui commence à lui porter préjudice ${ }^{3}$.

Malgré un rebond, l'année 1902 tend à prouver à la direction de la compagnie que la chute des bénéfices semble être irrémédiable. Le conseil d'administration désigne comme principale cause la concurrence française et étrangère ainsi que le ralentissement de l'activité industrielle. Cette concurrence semble définitivement installée dans le paysage industriel malgré les efforts consentis par la compagnie :

«Les baisses de prix que nous avons consenties au début de l'exercice n'ont pu réussir à écarter la concurrence étrangère et à ranimer la demande; dès le mois de janvier il a fallu imposer aux ouvriers des chômages périodiques (...)» (ADL, 1ETP3240, Mines de Montrambert : rapport du conseil d'administration à l'assemblée générale sur l'exercice 1902)

La direction des mines de Montrambert prend acte de cette concurrence qui va désormais la contraindre sur le marché des charbons de forge. Néanmoins, elle semble porter sa préoccupation sur les charbons secondaires qui à ses yeux constituent une opportunité de développement.

\section{2. .... au développement de la stratégie industrielle de la compagnie}

La crise industrielle qui frappe les mines de Montrambert n'est cependant pas une surprise pour le conseil d'administration. Celui-ci fait remarquer, en 1886, que l'ensemble des houillères stéphanoises tentent progressivement de transformer les produits livrés à la clientèle, dans le but de les améliorer ou d'en réduire le prix en faisant des qualités inférieures. Ce conseil émet l'idée de compléter les installations destinées à traiter les charbons. Il demande d'ailleurs l'ouverture d'une ligne budgétaire dans l'avenir afin d'édifier de nouvelles installations ${ }^{4}$. Dès 1903, la compagnie se

\footnotetext{
${ }^{1}$ Archives départementales de la Loire (désormais ADL), 1ETP3239, Mines de Montrambert : rapport du conseil d'administration à l'assemblée générale sur l'exercice 1883 (1884).

${ }^{2}$ ADL, 1ETP3239, Mines de Montrambert : rapport du conseil d'administration à l'assemblée générale sur l'exercice 1886 (1887).

${ }^{3}$ ADL, 1ETP3239, Mines de Montrambert : rapport du conseil d'administration à l'assemblée générale sur l'exercice 1894 (1895).

${ }^{4}$ ADL, 1ETP3239, Mines de Montrambert : rapport du conseil d'administration à l'assemblée générale sur l'exercice 1886 (1887).
} 
donne pour objectif de répondre à la demande des consommateurs qui apprécient de plus en plus les charbons de qualités secondaires ayant bénéficié d'un traitement adaptés.

A partir de 1895, les mines de Montrambert mettent en place une politique ambitieuse de valorisation des charbons secondaires en multipliant les installation de triage et de lavage. Les premiers résultats ne se font pas attendre. Cette politique prend une autre dimension lorsque le charbonnage décide en 1904 de s'attaquer au problème des déchets de la houille. La direction technique pense, à juste titre, que la réutilisation et la valorisation des déchets est possible, permettant ainsi la mise au point de nouveaux produits et de nouvelles sources d'énergie [ROJ 17].

Dès 1906, un gazogène d'essai est installé à côté des puits Dyèvre pour l'utilisation des pierres de triage contenant une forte proportion de combustible. Les premiers essais débutent rapidement et prouvent tout aussi rapidement qu'il est possible de produire un gaz à partir des déchets pouvant être employés dans des moteurs à explosion. Cette installation fait également l'objet d'autres expériences démontrant la possibilité de fabriquer des produits issus des déchets de la houille ${ }^{6}$.

Quelques mois avant ces essais, les houillères de Montrambert donnent pour mission en 1905 à l'un de ses ingénieurs, Francis Blache, d'observer les batteries de fours à coke édifiées par un mécanicien extérieur au charbonnage, $\mathrm{M}$. Lecoq. Ces fours ont la caractéristique de récupérer les déchets lors de la fabrication du coke ce qui intéresse grandement la houillère dans le cadre de son projet de mise en valeur des déchets. Blache doit également prendre contact avec Lecoq afin de lui soumettre certaines observations. Francis Blache doit aussi chiffrer les différentes solutions possibles pour l'édification d'un gazogène d'essai dérivé des fours à coke observés ${ }^{7}$. L'installation du gazogène d'essai est donc intimement liée à l'action de Blache. Les houillères de Montrambert par la mission confiée font de l'ingénieur le principal acteur chargé de la mise en œuvre de sa politique de diversification des produits issus de la houille.

Le choix de Francis Blache n'est cependant pas le fruit du hasard. Toutefois, nous connaissons peu de chose sur la vie personnelle de cet ingénieur : ses dates de naissance et de mort nous sont d'ailleurs inconnues. Il est probablement issu d'une famille de cultivateurs qui émigre dans la région stéphanoise au milieu du XIX ${ }^{\mathrm{e}}$ siècle. Seule sa formation à l'Ecole des mines de Saint-Étienne dans les deux dernières décennies du XIX ${ }^{\mathrm{e}}$ siècle apparaît comme un élément passé à la postérité. La formation qu'il reçoit au sein de cet établissement lui permet de s'intégrer dans le processus d'innovation que mettent en œuvre les mines de Montrambert depuis plusieurs décennies. Cette compagnie démontre depuis 1854 sa capacité à innover qui est en grande partie fondée sur les observations de ses ingénieurs lors des nombreux voyages d'études qu'ils effectuent à travers la France et l'Europe pour mettre au point des procédés techniques innovants [ROJ 17]. Ces ingénieurs possèdent une culture technique commune qu'ils ont acquise sur les bancs de l'Ecole des Mines. Anne-Françoise Garçon fait remarquer que la méthodologie à appliquer lors des voyages est transmise aux élèves à l'occasion des cours et des sorties de terrain. Ils sont invités à observer : les professeurs cherchent à éduquer le regard. Les élèves sont ainsi introduits mentalement dans l'atelier ou la mine recevant une description directe de ce qui s'y trouve, en même temps qu'ils apprennent à porter le regard là où il convient [GAR 03]. Les houillères de Montrambert ont également un lien très fort avec l'École des Mines par l'intermédiaire de son premier ingénieur principal Félix Devillaine qui devient rapidement directeur de la houillère jusqu'en 1895. Devillaine est issu de cet établissement formateur et est également président de l'Amicale des anciens élèves de l'école [ROJ 13]. Il met un point d'honneur à ne recruter à Montrambert que des ingénieurs issus de

\footnotetext{
${ }^{5}$ ADL, 1ETP3240, Mines de Montrambert : rapport du conseil d'administration à l'assemblée générale sur l'exercice 1902 (1903).

${ }^{6}$ ADL, 1ETP5789, Mines de Montrambert : rapport de l'ingénieur principal (1906-1908).
}

${ }^{7}$ ADL, 1ETP5831, Mines de Montrambert, traitement des charbons : note pour M. Blache (3 janvier 1905). 
l'établissement stéphanois. En 1895, Daniel Murgue succède à Devillaine à la tête des mines de Montrambert mais également quelques années plus tard à la tête de l'Amicale des anciens élèves. Ainsi il perpétue la logique de son prédécesseur en recrutant Francis Blache au début du $\mathrm{XX}^{\mathrm{e}}$ siècle.

La mission effectuée par Blache en 1905 et les expériences menées par le gazogène d'essai débouchent en 1909 sur la construction d'une usine destinée à utiliser les déchets de triage. Le conseil d'administration dévoile lors de l'assemblée générale de 1909 les premières ambitions de cette structure :

«(...) Après de longues études, nos ingénieurs ont commencé la construction de deux gazogènes d'essai, suivant un système pour lequel nous avons pris un brevet au nom de notre société, qui traiteront nos déchets et produiront le gaz utilisé dans des moteurs à explosion actionnant des alternateurs. Nous emploierons dans les services de la mine la force créée dans cette usine d'essai. Nous verrons ensuite, suivant les résultats obtenus, si nous devons vous proposer l'extension de cette installation, et la vente à des tiers de l'électricité produite. » (ADL, 1ETP3240, Mines de Montrambert : rapport du conseil d'administration à l'assemblée générale sur l'exercice 1909).

Cette usine qui est confiée à Francis Blache n'est pas encore un lieu de production mais un centre d'expérimentation que l'ingénieur se doit de faire évoluer pour satisfaire les besoins de l'entreprise et les exigences de sa hiérarchie.

\section{Prendre en main une usine d'expérimentation}

\subsection{L'alliance du laboratoire et de l'usine}

L'édification de l'usine d'essai débute en 1909 et s'achève en 1912. Celle-ci est située à proximité du centre administratif de la compagnie permettant à l'ingénieur principal et à la direction de conserver un regard sur l'évolution de cette structure. Dès 1913, l'usine est complétée par de nouveaux équipements. Cependant, il n'est pas question pour le charbonnage de la considérer comme un outil participant à la rentabilité de l'entreprise mais plutôt comme une installation en phase d'expérimentation ${ }^{8}$.

Francis Blache est donc chargé de mener à bien ces expérimentations et de résoudre le problème de l'utilisation des déchets de mine et des mauvais combustibles. Il affirme d'emblée sa méthode en mettant l'accent sur l'alliance entre le laboratoire et l'usine. Pour lui la solution passe par l'arrivée de la science dans le champ industriel [BLA 19]. Il est une fois encore dans la logique prônée par les ingénieurs issus de l'École des Mines de Saint-Étienne en plébiscitant la création d'une science industrielle, d'une science appliquée à l'industrie [ROJ 18]. Sa démarche correspond à cette logique. En effet, au début de ses expérimentations Blache part d'un postulat clairement établi :

«On peut poser, à mon avis, comme principe général, que l'utilisation industrielle des combustibles est un problème d'ordre thermo-chimique et que toute installation de quelque importance doit être conçue dans le but de récupérer tous les produits utilisables de leur transformation. » (Blache, «Utilisation des déchets de mines et des mauvais combustible », Bulletin de la société de lindustrie minérale, 1919)

C'est ce postulat qui l'amène à choisir le gazogène pour résoudre le problème dont il a la charge. En effet, pour Blache le gazogène se présente comme un véritable laboratoire où tous les corps en présence réagissent en produisant des combinaisons chimiques et calorifiques pouvant lui permettre d'obtenir le résultat que la compagnie ambitionne.

\footnotetext{
${ }^{8}$ ADL, 1ETP3240, Mines de Montrambert : rapport du conseil d'administration à l'assemblée générale sur l'exercice 1912 et 1913 
L'installation de l'usine en 1912 n'est pas la fin du processus d'expérimentation mais le début. Comme le fait remarquer l'ingénieur principal, la mise à feu de l'usine soulève de nombreux problèmes : la question de la séparation des goudrons, la séparation des sous-produits ammoniacaux, la récupération des sous-produits...... . . Des dysfonctionnements apparaissent donc au fil de l'utilisation de l'usine. Ce sont ces dysfonctionnements qui permettent à Blache de cibler les problèmes à résoudre et d'orienter son action vers l'épuration des gaz [BLA 19].

Dans cette tâche, Francis Blache est assisté par M. Beaudequin un ingénieur constructeur de gazogène mais également par un chimiste dont l'activité est intégralement dédiée à l'usine d'essai ${ }^{10}$. Les analyses effectuées par cette équipe permettent donc d'identifier les différents problèmes à résoudre. Cependant les solutions expérimentées ne sont pas le fruit du hasard mais d'un important travail de veille technologique assuré par Francis Blache lui-même. En effet, lorsqu'un problème est ciblé, Blache n'hésite pas à effectuer des voyages d'étude afin de rechercher des sources d'inspiration. Ainsi dès 1913, il se rend à l'usine électrique de la Compagnie d'Orléans à Tours pour observer leurs gazogènes «Richée ». Ce voyage lui permet d'étudier un principe qui s'avère très judicieux en matière de dégoudronnage ${ }^{11}$. Les premiers déplacements de Blache, en 1913 et 1914, sont généralement des prises d'informations sur les gazogènes employés par d'autres industries. Mais dans les années 1920, les voyages deviennent de véritables recherches de solutions à un problème très précis à l'image de sa visite effectuée à l'usine à gaz de Grenoble le 26 août $1924^{12}$. L'objectif est ici de se renseigner sur le procédé de débenzolage du gaz par voie sèche qui y est appliqué. Il n'est cependant pas question pour lui de se contenter d'une seule source d'inspiration lorsqu'un problème est à résoudre. Ainsi il complète son voyage à Grenoble par une visite à l'usine à gaz de Chambéry pour observer l'installation de débenzolage ${ }^{13}$.

Le perfectionnement technique des procédés de production occupe donc Blache et son équipe durant les premières années d'existence de l'usine. Il n'est pas question encore d'une production rentable intégrant une logique industrielle et économique mais l'ingénieur entrevoit durant ces premières années certains marchés que la compagnie pourrait investir.

\subsection{Apercevoir les marchés potentiels}

L'application de la science à l'industrie et plus précisément à la transformation des charbons constitue pour Francis Blache une opportunité de fournir à l'industrie de nouvelles matières premières ainsi que de nouveaux carburants [REU 24]. Autrement dit, la science est pour lui un vecteur pouvant générer de nouveaux marchés. D'ailleurs dès les premières années à la tête de l'usine d'essai lorsque celle-ci commence à produire du gaz, Blache envisage de développer le procédé pour créer des centrales à gaz et/ou électricité pour alimenter d'autres industries. Il imagine également les usages domestiques en distribuant directement par des réseaux le gaz produit faisant disparaître l'emploi direct du combustible dans les foyers domestiques [BLA 19]. Les réflexions de l'ingénieur de Montrambert sont en adéquation avec son époque et les marchés économiques naissants. En effet, dans les premières décennies du $X X X^{\mathrm{e}}$ siècle des sociétés de production d'électricité et de gaz construisent des centrales en ville, puis en banlieue, afin de fournir les foyers urbains qui commencent à s'équiper en réseau de distribution de gaz et d'électricité [WOR 98].

\footnotetext{
${ }^{9}$ ADL, 1ETP5790, Mines de Montrambert : rapport de l’ingénieur principal (1912).

${ }^{10}$ ADL, 1ETP5790, Mines de Montrambert : rapport de l'ingénieur principal (1912).

11 ADL, 1ETP3269, Mines de Montrambert, Voyages d'étude: Note sur les gazogènes « Richée » en service à l'usine de la Compagnie d'Orléans à Tours (2 juillet 1913).
}

${ }^{12}$ ADL, 1ETP3269, Mines de Montrambert, Voyages d'étude : visite à l'usine à gaz de Grenoble (26 août 1924).

${ }^{13}$ ADL, 1ETP3269, Mines de Montrambert, Voyages d'étude : visite à l'usine à gaz de Chambéry (12 septembre 1924). 
Si les industriels tendent à développer des produits leur permettant d'élargir leur marché, le contexte de l'époque peut donner une accélération non négligeable à leur dessein. En effet, la Première Guerre mondiale et la mobilisation de l'industrie qu'elle provoque pousse les acteurs de l'industrie à s'intéresser aux possibilités nouvelles qui s'offrent à eux en matière d'approvisionnement notamment dans le domaine sidérurgique. Ainsi, le Bulletin des usines de guerre créé par le gouvernement français pour informer les industriels sur les innovations à mettre en œuvre durant le conflit met en avant le travail de Francis Blache à Montrambert. Cette publication officielle destinée aux industriels insiste sur les services que peuvent rendre les gazogènes employés par la houillère stéphanoise: production de vapeur dans les chaudières munies de chambres de combustion, chauffage des fours métallurgiques, éclairage des villes et production de force motrice [GOU 18].

Le conflit a mis en lumière l'installation conçue par Blache et dès 1919 les retombées semblent s'annoncer. Le monde de l'industrie a pris connaissance de l'innovation et manifeste un intérêt que le journal le Messager économique français relaie dans son édition du 9 février 1919 :

«La question de l'utilisation des combustibles pauvres, des déchets de triage et de lavage vient d'être résolue par cette société. Ces combustibles sont d'abord traités au four à coke avec récupération des sous-produits, les cokes sont ensuite traités au gazogène. La société récupère par tonne de charbon : $27 \mathrm{Kg}$ de goudron, $12 \mathrm{Kg}$ de sulfate d'ammoniaque. En outre, le traitement au gazogène de 1,5 $\mathrm{K}$ donne 1 Kilowatt au moteur à gaz. Malgré des installations sommaires, la société arrive à produire mensuellement 250000 Kilowatts-heure. La plus grande partie est absorbée par les services de la mine. L'excédent est pris par le réseau de l'Electrique de la Loire et du Centre.» (Messager économique français, 9 février 1919).

Les voyages d'étude effectués par Blache sont également pour lui un moment de découverte de certains marchés potentiels auxquels il ne pense pas. Ainsi, lorsqu'il visite les usines Arbel à Rivede-Gier en octobre 1922, il remarque que l'entreprise emploie des camions fonctionnant avec un gazogène à gaz pauvre alimenté par du bois ou du charbon de bois. A cet instant Blache prend conscience d'un marché celui du transport dans lequel les mines de Montrambert pourraient s'engouffrer en fournissant le gaz pauvre produit par son usine. Il note que plusieurs entreprises commencent à investir dans cette solution de transport :

«En résumé, l'emploi du gazogène à gaz pauvre paraît réaliser une solution économique du transport sur route. La société de Vierzon a commandé 500 camions pour la Pologne où le bois est particulièrement abondant. » (ADL, 1ETP3269, Mines de Montrambert, Voyages d'étude : visite aux usines Arbel 20 octobre 1922).

De nouveaux marchés semblent s'ouvrir à Montrambert grâce à l'apport de l'usine d'essai. Celle-ci va rapidement dans les années 1920 se transformer en usine de production et ainsi jouer un véritable rôle économique et industriel au sein de la houillère.

\section{Diriger des usines de production}

\subsection{Rendre l'expérimentation économiquement viable}

L'usine dirigée par Francis Blache ne passe pas brutalement du statut d'unité d'expérimentation à celui de lieu de production. Si ce glissement est progressif, il semble que l'après Première Guerre mondiale marque une stabilisation dans le processus de traitement des déchets de la houille et des mauvais combustibles. Désormais les deux phases du traitement sont clairement identifiées et maîtrisées : carbonisation et gazéification. Cela permet à la houillère de produire $2,5 \mathrm{~m}^{3}$ de gaz par kilogramme de combustible. Ce traitement des déchets produit également 12 à 14 kilogrammes de sulfate d'ammoniaque par tonne de déchets, 30 kilogrammes de goudron ainsi que de l'énergie électrique consommée par les services du charbonnage ou revendue à la Compagnie électrique de la 
Ainsi les premiers sous-produits industriellement exploitables permettent de fixer l'organisation technique de l'usine. Celle-ci est désormais responsable des fours à coke pour produire le coke métallurgique mais elle est aussi équipée d'un système de gazogène. Au fil des années l'installation se développe et il n'est plus question de l'usine mais des usines : un atelier de rectification des benzols apparaît, les usines se dotent d'un mélangeur à mourre automatisé.... Plus généralement ces unités de production se mécanisent et s'automatisent au début des années 1930. L'objectif est clairement annoncé par Francis Blache dans son rapport annuel de 1930 : faire chuter le prix de revient des sous-produits ${ }^{14}$.

La préoccupation économique prend le pas sur la stricte dimension technique. Il n'est plus uniquement question de mettre au point des procédés capables de fabriquer de nouveaux produits. Ceux-ci sont désormais regardés à l'aune du prix de revient et plus globalement du bénéfice qu'ils peuvent générer. Les voyages d'étude menés par Blache et les membres de son équipe sont à cet égard significatifs. La pratique du voyage destinée à solutionner un problème perdure mais les observations évoluent quelque peu. En effet, Blache ou les ingénieurs qu'il mandate prennent en compte de nouveaux aspects lorsqu'ils décrivent et analysent des procédés industriels. Ainsi la fiabilité des installations devient un critère central à l'image de la visite effectuée en octobre 1924 en Belgique pour observer les fours à coke de Moll ${ }^{15}$. Cette idée de régularité et de sûreté dans le fonctionnement est une donnée essentielle pour assurer un prix de revient peu élevé et offrir une compétitivité des produits du charbonnage sur les marchés des sous-produits de la houille. Toutes les visites effectuées sont dorénavant guidées par la recherche du procédé technique générant le prix de revient le plus faible possible. Lorsque Blache se rend à la société chimique de Gerland en mars 1926 pour observer les moyens de régénérer l'huile usagée ayant servi au débenzolage du gaz, il a déjà effectué plusieurs visite de ce type, l'objectif étant de :

«L'étude de ces différents constructeurs nous fixera sur le choix des appareils à installer qui nous paraîtront les plus efficaces et les moins coûteux. »(ADL, 1ETP3269, Mines de Montrambert, Voyages d'étude : visite à la société chimique de Gerland 12 mars 1926)

Les voyages de veille technologique sont également révélateurs des collaborations techniques et industrielles existantes entre les différentes houillères. En effet, Blache ne visite pas uniquement que des usines mais aussi des charbonnages. Ces concurrents n'hésitent pas à lui ouvrir leur porte afin d'échanger sur la fabrication des sous-produits de la houille. Ainsi, en juin 1923, l'ingénieur de Montrambert se rend aux mines de Béthune pour observer la conception de l'AZ $\mathrm{H}^{3}$ synthétique, un gaz qu'il souhaite fabriquer à partir de l'hydrogène contenu dans les gaz des fours à coke. Une fois encore, il fixe son attention sur le procédé technique mais aussi sur les bénéfices engendrés par l'installation ${ }^{16}$.

Un laboratoire embryonnaire apparaît dans l'usine à la fin de la Première Guerre mondiale et prend une dimension centrale à la fin des années 1920. Il devient la pierre angulaire de l'organisation des usines en assurant le contrôle et les essais. Ainsi, il expérimente de nouveaux procédés et met au point de nouveaux produits qui, à terme, sont fabriqués et commercialisés par le charbonnage. Dès 1930 l'activité du laboratoire est immense. Il mène des analyses sur les charbons, les cokes, les scories des chaudières. Il effectue également des essais sur les poussières de mine, sur les différents cokes, sur l'élimination $\mathrm{du}$ soufre des benzols.... Toutes ces études aboutissent à des expérimentations grandeur nature à l'image des essais qui ont lieu à l'atelier de rectification des benzols sur la question de l'élimination du soufre des benzols. A travers l'activité du laboratoire,

\footnotetext{
${ }^{14}$ ADL, 1ETP5802, Mines de Montrambert, usines : rapports 1930-1931.

${ }^{15}$ ADL, 1ETP3270, Mines de Montrambert, Voyages d'étude : visite aux fours à coke de Moll (Belgique) (29 octobre 1924).

${ }^{16}$ ADL, 1ETP3269, Mines de Montrambert, Voyages d'étude : visite de l'installation de la compagnie des mines de Béthune pour la fabrication de l'AZH ${ }^{3}$ synthétique (2 juin 1923).
} 
Blache met en œuvre son idée initiale au service des intérêts économiques de la houillère : l'alliance du laboratoire et de l'usine.

\subsection{Accéder à de nouveaux marchés et offrir un second souffle à la houillère}

Á la fin des années 1920, les usines sont en mesure de fabriquer de nombreux sous-produits. Elles travaillent notamment à la récupération de nombreuses substances issues des déchets de la houille : récupération de l'ammoniaque par condensation et lavage, atelier à sulfate, récupération des benzols ${ }^{17}$. Si ces sous-produits trouvent un écoulement aisé sur les marchés, c'est surtout la production de gaz qui offre à la compagnie des perspectives économiques intéressantes. Dès 1933, Blache note que les ventes de gaz augmentent de plus de $15 \%$ par rapport à l'année précédente. Montrambert fournit de nombreuses entreprises locales comme la Société de l'Energie industrielle, la Compagnie du gaz du midi ou encore la Compagnie de gaz de Saint-Étienne. D'ailleurs le directeur des usines note en 1933 :

«Les sous-produits de la récupération trouvent un écoulement facile et rémunérateur ; mais c'est surtout le développement des ventes de gaz qui peut permettre d'accroître, d'une façon particulièrement intéressante, la marge des bénéfices. »(ADL, 1ETP5802, Mines de Montrambert, usines : rapport 1933)

Cette politique gazière permet donc à la houillère de fournir des entreprises qui n'étaient pas auparavant des clients. Les sociétés distribuant le gaz dans les centres urbains constituent des débouchés nouveaux et importants comme la Société du gaz de Firminy ou la Compagnie du Bourbonnais. La quantité de gaz fournie à ces entreprises est en constante augmentation au début des années 1930. Ainsi les émissions de gaz sur le réseau de la Société du gaz de Firminy s'accroissent de près de $10 \%$ en $1930^{18}$. D'autres entreprises achètent les gaz pauvres de Montrambert pour faire fonctionner des moteurs comme les établissements métallurgiques Jacquemard consommant près de $650000 \mathrm{~m}^{3}$ de gaz ou encore la verrerie Souchon-Neuvesel qui fait fonctionner son usine au $\mathrm{gaz}^{19}$.

Les sous-produits issus de la récupération sont rapidement commercialisés. Dès 1922, le goudron, le sulfate d' $\mathrm{AZH}^{3}$ et les benzols offrent à Montrambert un bénéfice de 749335 francs ${ }^{20}$. Cependant, il ne s'agit que d'un appoint assez irrégulier contrairement aux débouchés gaziers. Néanmoins, le laboratoire œuvre pour répondre aux demandes des différents marchés. Il tente notamment de mettre au point un coke spécial à partir des déchets de la houille qui semble très apprécié sur le marché italien.

Au-delà des marchés liés aux entreprises, les mines de Montrambert se préoccupent également des particuliers. Depuis sa création en 1854 , le charbonnage propose à la vente du charbon pour le chauffage domestique. Cependant celui-ci est d'une grande qualité et les conditions de son extraction nécessite un investissement notable donnant ainsi un prix de revient et conséquemment de vente assez élevé. Cette clientèle s'accroît depuis plusieurs années, Francis Blache en est d'ailleurs conscient et c'est pour lui un enjeu de développement :

«La clientèle de chauffage domestique, tend à se développer. Notre coke est un combustible bien adapté aux emplois domestiques. L'amélioration de la qualité de notre coke, peut-être trouvée par

\footnotetext{
${ }^{17}$ ADL, 1ETP5802, Mines de Montrambert, usines : rapport 1930.

${ }^{18}$ ADL, 1ETP5802, Mines de Montrambert, usine : rapport 1930.

${ }^{19}$ ADL, 1ETP5836, Mines de Montrambert, service commercial : lettre du directeur de la verrerie Souchon-Neuvesel à André

Pigeot (directeur e Montrambert) (19 septembre 1922).

${ }^{20}$ ADL, 1ETP5791, Mines de Montrambert, rapport de l’ingénieur principal (1922).
} 
l'emploi de fines 0.2 ou $0.3 \mathrm{~mm}(. .) ».($ ADL, 1ETP5802, Mines de Montrambert, usine : rapport 1931)

Le laboratoire répond rapidement à la volonté de son directeur en mettant au point à partir du coke produit et des déchets de la houille un sous-produit destiné au marché du chauffage domestique : le Monanthra. A la suite de nombreux essais qui se montrent concluants, des batteries de fours à gazogène sont dédiées à cette fabrication. Celle-ci est très active durant les mois d'hiver notamment en janvier, février et mars alors que durant les mois estivaux la production est stoppée. En effet, il est déjà difficile à cette époque de l'année d'écouler le surplus de la période hivernale ${ }^{21}$.

Ainsi les usines prennent, à partir des années 1920, une place prépondérante dans la politique économique de la houillère devenant plus qu'une simple unité d'expérimentation. La position personnelle de Francis Blache révèle d'ailleurs cet état de fait. S'il est nommé simple responsable de l'usine d'essai en 1909 lors de son édification, il devient en 1921 ingénieur principal des usines soit le troisième personnage le plus important du charbonnage derrière le directeur et l'ingénieur principal $^{22}$. La place désormais occupée par Blache dans l'organigramme démontre l'importance des usines au sein des mines de Montrambert et de la Béraudière.

\section{Conclusion}

Le contexte économique et industriel de la fin du $\mathrm{XIX}^{\mathrm{e}}$ siècle remet en cause la prospérité que les charbonnages connaissent depuis plusieurs décennies. A la crise métallurgique succède le début d'une mutation du système énergétique avec l'utilisation de plus en plus importante de l'électricité. Ces phénomènes successifs poussent les houillères à réagir. Les mines de Montrambert et de la Béraudière parient sur l'innovation pour conquérir de nouveaux marchés. L'expérimentation est donc au cœur de leur action qu'elles confient à Francis Blache un ingénieur recruté quelques années auparavant.

Le parcours de ce personnage éclaire le positionnement de l'innovateur au sein de l'entreprise. Loin de la figure omnipotente du génial inventeur, Blache doit répondre aux exigences de sa hiérarchie. S'il n'est pas responsable de l'objectif à atteindre, il est cependant garant du chemin emprunté en choisissant l'alliance du laboratoire et de l'usine. Le choix effectué par la houillère n'est cependant pas anodin, le conseil d'administration nomme à la tête de l'usine d'essai un ingénieur pouvant s'intégrer dans la culture de l'innovation de l'entreprise. La figure de l'innovateur solitaire est également remise en cause par le parcours de Francis Blache qui est entouré par de nombreux autres ingénieurs participant au travail de veille technologique. Une autre équipe, composée d'ingénieurs et de chimistes, l'assiste au sein du laboratoire des usines qu'il dirige. Ainsi l'innovation n'est plus une œuvre solitaire mais collective.

Le processus d'innovation mis en œuvre par Francis Blache met également en lumière la convergence des sciences et des techniques. Autrement dit la place de plus en plus importante prise par la science dans l'industrie par le biais du laboratoire. Si celui-ci s'impose dans certaines usines métallurgiques au cours du derniers tiers du XIX $\mathrm{X}^{\mathrm{e}}$ siècle, c'est avec la fin de la Première Guerre mondiale que le laboratoire industriel va se répandre massivement dans les mines et les usines métallurgiques à l'image des usines d'expérimentation des mines de Montrambert.

Enfin, ce parcours nous laisse entrevoir l'influence du contexte sur le phénomène d'innovation. Dans le cas présent la Première Guerre mondiale joue un rôle de catalyseur et d'accélérateur en focalisant l'attention des milieux industriels sur les innovations mis en place par Francis Blache à Montrambert.

\footnotetext{
${ }^{21}$ ADL, 1ETP5802, Mines de Montrambert, usine : rapports1933-34.

${ }^{22}$ ADL, 1ETP4539, Mines de Montrambert, appointement des ingénieurs : tableau d'appointement (22 avril 1921). 


\section{Bibliographie}

\section{Ouvrages}

[BER 07] BERGSON H., L'évolution créatrice, Presses Universitaires de France, Paris, 1907 (réed 1941).

[CAR 97] CARON F., Les deux révolutions industrielles du XXe siècle, Agora, Paris, 1997.

[GAR 03] GARÇON A-F., Entre l'Etat et l'usine. L'Ecole des Mines de Saint-Etienne au XIXe siècle, Presses Universitaires de Rennes, Rennes, 2003.

[GRA 08] GRAS L-J., Histoire économique de la métallurgie de la Loire, imprimerie Théolier, Saint-Étienne, 1908.

[GRA 22] GRAS L-J., Histoire économique générale des mines de la Loire, imprimerie Théolier, Saint-Étienne, tome 2, 1922.

[ROJ 08] ROJAS L., Histoire de révolution technologique. De l'exploitation artisanale à la grande industrie houillère de la Loire, L'Harmattan, Paris, 2008.

[ROJ 17] ROJAS L., l'intelligence technique des ingénieurs civils des mines. Pratiques techniques des ingénieurs des houillères de Montrambert et de la Béraudière (1/854-1945), Editions universitaires européennes, Saarbrücken, 2017.

[VEY 99] VEYRON T., L'évolution technique des houillères françaises et belges 1800-1880, L'Harmattan, Paris, 1999.

[WOR 98] WORONOFF D., Histoire de l'industrie en France, Le Seuil, Paris, 1998.

\section{Articles de revue}

[BLA 19] BLACHE F., «Utilisation des déchets de mines et des mauvais combustibles », Bulletin de la société de l'industrie minérale, p. 5-28, 1919.

[BLA 20] BLACHE F., «L'utilisation des combustibles », La houille blanche, p. 152-155, 1920.

[GOU 18] GOUVY A., « Utilisation des gaz et autres sous-produits dans l'industrie sidérurgique », Bulletin des usines de guerre, $\mathrm{n}^{\circ} 11$, p. 1-3, 8 juillet 1918.

[REU 24] REUNION DU DISTRICT DE SAINT-ÉTIENNE, « compte-rendu de la réunion du 12 janvier 1924 », Revue de l'industrie minérale, $\mathrm{n}^{\circ} 78,1924$.

[ROJ 13] ROJAS L., «Félix Devillaine (1823-1913) ou la figure de l'ingénieur praticien », Cahier de RECITS, n9, p. 95-111, 2013.

[ROJ 18] ROJAS L., «Construire et diffuser le savoir des ingénieurs civils des mines : le Bulletin de la société de l’industrie minérale (1855-1914)», Philosophia Scientiae, Vol. 22 cahier 1, p. 185-201, 2018. 